\title{
TWO-ELEMENT GENERATION OF THE SYMPLECTIC GROUP(1)
}

\author{
BY
}

\section{PETER STANEK}

1. Introduction. Several authors have discussed the problem of finding pairs of generators for the known simple groups of finite, composite order (see $[1 ; 2$; $7 ; 8 ; 9])$. In this paper we examine the symplectic group, the group of all $2 n$ by $2 n$ matrices, $X$, with entries from $\mathrm{GF}(q)$, which satisfy

$$
X H X^{T}=H, \quad H=\left(\begin{array}{rr}
0 & I \\
-I & 0
\end{array}\right), \quad X^{T}=X \text { transpose }
$$

0 and $I$ the zero and identity matrices, respectively. We denote this group by $\operatorname{Sp}(2 n, q)$, and by $\operatorname{PSp}(2 n, q)$ the factor group of $\operatorname{Sp}(2 n, q)$ by its center, $\{ \pm I\}$. $\operatorname{PSp}(2 n, q)$ is a simple group of order

$$
\frac{1}{d} q^{n^{2}} \prod_{i=1}^{n}\left(q^{2 i}-1\right)
$$

$d$ the g.c.d. of 2 and $q-1$, except for $\operatorname{PSp}(2,2)=S_{3}, \operatorname{PSp}(2,3)=A_{4}, \operatorname{PSp}(4,2)=S_{6}$ (see $[4 ; 5])$. We prove

THEOREM. The group $\operatorname{PSp}(2 n, q)$, for $n \geqq 3$, has two generators, one of period (group order) two.

In [1] the corresponding result is proved for the projective unimodular group. In [8] it is proved that $\operatorname{PSp}(2 n, q), q$ a prime, is generated by two of its elements, while in [9] this is proved for all of the known simple finite groups other than the alternating and Mathieu groups.

For $q=2$, T. G. Room [7], has proved this theorem. The result has certain geometric implications [3].

2. Known generators of the symplectic group. The following is originally due to Dickson [4]. The form in which we state it is convenient for our purposes; and, for a proof, that of Hua and Reiner [6], may be easily modified.

LEMMA 1. $\operatorname{Sp}(2 n, q)$ is generated by the following matrices:

\section{(i) translations}

Received by the editors August 21, 1962.

(1) This work is in part an excerpt from the author's doctoral thesis, supported by NSF grant G-9504. I am greatly indebted to Professor A. A. Albert for suggesting the problem and giving valuable criticism. 


$$
T=\left(\begin{array}{cc}
I & S \\
0 & I
\end{array}\right), \quad S^{T}=S
$$

(ii) rotations

$$
R=\left(\begin{array}{cc}
U & 0 \\
0 & \left(U^{T}\right)^{-1}
\end{array}\right), \quad \operatorname{det} U \neq 0
$$

(iii) semi-involutions

$$
S=\left(\begin{array}{rr}
Q & I-Q \\
Q-I & Q
\end{array}\right)
$$

where $Q$ is a diagonal matrix of zeros and ones.

In all of what follows, $E_{i j}$ will be the $n$ by $n$ matrix with a one in the $i j$ th position and zero elsewhere.

For $x \in \mathrm{GF}(q)$, define

$$
\begin{aligned}
T_{i}(x) & =\left(\begin{array}{ll}
I & x E_{i i} \\
0 & I
\end{array}\right) ; \\
T_{i j}(x) & =\left(\begin{array}{cc}
I & x E_{i j}+x E_{j i} \\
0 & I
\end{array}\right) ; \\
R_{i j}(x) & =\left(\begin{array}{cc}
I+x E_{i j} & 0 \\
0 & I-x E_{j i}
\end{array}\right) ;
\end{aligned}
$$

for $i \neq j$. The $T$ 's commute, while the rotations satisfy $\left(R_{i j}(x)\right)^{-1}=R_{i j}(-x)$, $\left(R_{i j}(x), R_{j k}(y)\right)=R_{i k}(x y)$ if $i \neq k \neq j$, where $(U, V)$ is the commutator $U V U^{-1} V^{-1}$.

3. $n \geqq 3$ and $q$ odd.

LEMMA 2. $\operatorname{Sp}(2 n, q)$ is generated by

$$
D=\left[\begin{array}{cc}
\sum_{i=1}^{n-1} E_{i, i+1} & -E_{n 1} \\
E_{n 1} & \sum_{i=1}^{n-1} E_{i, i+1}
\end{array}\right]
$$

and $\mathbf{J}^{\prime}=R_{21}(a)$, where $a$ is primitive in $\mathrm{GF}(q)$.

Clearly $D$ and $J^{\prime}$ belong to $\operatorname{Sp}(2 n, q)$. Consider the conjugates of $J^{\prime}$ by $D$ :

$$
\begin{aligned}
D^{-1} J^{\prime} D & =R_{32}(a) \\
D^{-1} R_{32}(a) D & =R_{43}(a) \\
& \vdots \\
D^{-1} R_{n, n-1}(a) D & =\left(T_{n 1}(a)\right)^{T} \\
D^{-1}\left(T_{n 1}(a)\right)^{T} D & =R_{12}(-a) \\
& \vdots \\
D^{-1} R_{n-1, n}(-a) D & =T_{n 1}(-a) \\
D^{-1} T_{n 1}(-a) D & =R_{21}(a) .
\end{aligned}
$$


Since $R_{i j}(-x)=\left(R_{i j}(x)\right)^{-1}$, the group generated by $D$ and $J^{\prime}$ contains every rotation of the form $R_{i, i+1}( \pm a)$ and $R_{i+1, i}( \pm a)$, for all possible values of $i$.

(3)

$$
\begin{aligned}
\left(R_{12}(a), R_{23}(a)\right) & =R_{13}\left(a^{2}\right) \\
\left(R_{13}\left(a^{2}\right), R_{32}(a)\right) & =R_{12}\left(a^{3}\right) \\
& \vdots \\
\left(R_{12}\left(a^{2 j+1}\right), R_{23}(a)\right) & =R_{13}\left(a^{2 j+2}\right) \\
\left(R_{13}\left(a^{2 i}\right), R_{32}(a)\right) & =R_{12}\left(a^{2 i+1}\right),
\end{aligned}
$$

so that every $R_{13}\left(a^{2 i}\right)$ and $R_{12}\left(a^{2 j+1}\right)$, for every integer value of $i$ and $j$, is obtained. Now $q$ is odd, so $q-1$ is even and we have $R_{13}(1)$. But there exist in $\mathrm{GF}(q)$ solutions $x, y$ of $x^{2}+y^{2}=a^{-1}$. Let $x=a^{i}$ and $y=a^{j}$; then $x^{2}=a^{2 i}$, $y^{2}=a^{2 j}$ and

$$
R_{13}\left(a^{2 i}\right) R_{13}\left(a^{2 j}\right)=R_{13}\left(x^{2}+y^{2}\right)=R_{13}\left(a^{-1}\right)
$$

So

$$
\left(R_{13}\left(a^{-1}\right), R_{32}(a)\right)=R_{12}(1)
$$

is available.

By replacing $a$ by 1 in (2), we see that the conjugates of $R_{12}(1)$ under $D$ contain every $R_{i, i+1}(1)$ and $R_{i+1}(1)$. Then

$$
\begin{aligned}
\left(R_{12}(1), R_{23}(1)\right) & =R_{13}(1) \\
\left(R_{13}(1), R_{34}(1)\right) & =R_{14}(1) \\
& \vdots \\
\left(R_{1 i}(1), R_{i, i+1}(1)\right) & =R_{1, i+1}(1) \\
& \vdots \\
\left(R_{32}(1), R_{21}(1)\right) & =R_{31}(1) \\
& \vdots \\
\left(R_{i+1, i}(1), R_{i, 1}(1)\right) & =R_{i+1,1}(1)
\end{aligned}
$$

so that we get every $R_{i 1}(1)$ and $R_{1 j}(1)$, for all possible $i$ and $j$.

Now let $i \neq j$. If $i \neq 1 \neq j$,

$$
\left(R_{i 1}(1), R_{1 j}(1)\right)=R_{i j}(1)
$$

and since

$$
\left(R_{13}\left(a^{2 k}\right), R_{32}(1)\right)=R_{12}\left(a^{2 k}\right)
$$

then,

$$
\begin{aligned}
& \left(R_{i 1}(1), R_{12}(u)\right)=R_{i 2}(u), \quad i \neq 2 \\
& \left(R_{i 2}(u), R_{2 k}(1)\right)=R_{i k}(u), \quad i \neq k \neq 2 .
\end{aligned}
$$


We know that every $n$ by $n$ matrix, $U$, of determinant one can be written as a product of matrices of the form $I+x E_{i j}$. Hence, the group generated by $D$ and $J^{\prime}$ contains every rotation (ii) with $\operatorname{det} U=1$.

Now,

$$
\begin{aligned}
D^{-1} R_{1 n}(x) D & =T_{12}(x), \\
\left(R_{12}\left(\frac{1}{2}\right), T_{12}(x)\right) & =T_{1}(x),
\end{aligned}
$$

and we get every $T_{1}(x)$ and every $T_{12}(x)$. Also, $\left(T_{1}(x)\right)^{T}=D^{-n-1} T_{1}(-x) D^{n+1}$. If $S_{1}(x)=T_{1}(x)\left(T_{1}\left(-x^{-1}\right)\right)^{T} T_{1}(x)$, then

$$
S_{1}(-a) S_{1}(1)=\left(\begin{array}{cc}
I-E_{11}+a E_{11} & 0 \\
0 & I-E_{11}+a^{-1} E_{11}
\end{array}\right) .
$$

Every $n$ by $n$ matrix of nonzero determinant is a product of a matrix of determinant one and a matrix $I-E_{11}+x E_{11}, x \neq 0$ in $\mathrm{GF}(q)$. Since $a$ is primitive, every element in $\operatorname{GF}(q)$ is some power of $a$, and we see that the group generated by $D$ and $J^{\prime}$ contains every matrix of the form (ii), with $\operatorname{det} U \neq 0$.

We have obtained the matrices $T_{1}(x)$ and $T_{12}(x)$ from $D$ and $J^{\prime}$. Since

$$
\begin{aligned}
\left(\begin{array}{ll}
I & S \\
0 & I
\end{array}\right) \quad\left(\begin{array}{ll}
I & S^{\prime} \\
0 & I
\end{array}\right) & =\left(\begin{array}{ll}
I & S+S^{\prime} \\
0 & I
\end{array}\right), \\
\left(\begin{array}{cc}
U & 0 \\
0 & \left(U^{T}\right)^{-1}
\end{array}\right)\left(\begin{array}{ll}
I & S \\
0 & I
\end{array}\right) \quad\left(\begin{array}{cc}
U^{-1} & 0 \\
0 & U^{T}
\end{array}\right) & =\left(\begin{array}{cc}
I & U S U^{T} \\
0 & I
\end{array}\right),
\end{aligned}
$$

we see that every translation can be obtained by simultaneously interchanging rows and corresponding columns of the symmetric matrices $x E_{11}$ and $x E_{12}+x E_{21}$ of $T_{1}(x)$ and $T_{12}(x)$, respectively, and then taking their products.

Now define

$$
S_{i, j, k, \ldots}=\left(\begin{array}{cc}
Q & I-Q \\
Q-I & Q
\end{array}\right)
$$

where $Q$ has zeros in the $i i t h, j j$ th, $k k$ th, $\cdots$ positions, and ones in all other diagonal positions. Then $S_{2}, \cdots, S_{n}$ are among the conjugates of $S_{1}$ under $D$; and since

$$
\left(S_{i, j, k, \ldots}\right)\left(S_{i_{1}, j_{1}, k_{1}, \ldots}\right)=S_{i, j, k}, \ldots, i_{1}, j_{1}, k_{1}, \ldots
$$

we see that every generator of the group $\operatorname{Sp}(2 n, q)$ can be obtained from $D$ and $J^{\prime}$ for $n>2$ and $q$ odd. We return now to the case of characteristic two.

\section{4. $n \geqq 4$ and $q$ a power of two.}

LEMMA 3. $\operatorname{Sp}\left(2 n, 2^{m}\right)$ is generated by the matrix D of Lemma 2 and $J^{\prime}$ :

$$
J^{\prime}=\left(\begin{array}{cc}
I+a E_{21} & E_{n n} \\
0 & I+a E_{12}
\end{array}\right),
$$


a primitive in $\mathrm{GF}(q)$.

$$
\begin{aligned}
D^{-1} J^{\prime} D & =\left(\begin{array}{cc}
I+a E_{32} & 0 \\
E_{11} & I+a E_{12}
\end{array}\right)=B . \\
\left(J^{\prime}, B\right) & =R_{31}\left(a^{2}\right) . \\
\left(J^{\prime}, R_{31}\left(a^{2}\right)\right) & =R_{23}\left(a^{3}\right) .
\end{aligned}
$$

Notice that for $i_{0}$ and $j_{0}$ fixed, the conjugates of $R_{i_{0} j_{0}}(x)$ under $D$ (see (2)) contain the matrices $R_{s t}(x)$, where $|s-t|=\left|i_{0}-j_{0}\right|$.

$$
\begin{aligned}
& \left(R_{23}\left(a^{3}\right), R_{31}\left(a^{2}\right)\right)=R_{21}\left(a^{5}\right) \\
& \left(R_{21}\left(a^{5}\right), R_{13}\left(a^{2}\right)\right)=R_{23}\left(a^{7}\right)
\end{aligned}
$$

Now $a^{q-1}=1$ is an odd power of $a$, so we obtain from $D$ and $J^{\prime}$ every $R_{i, i+1}(1)$ and $R_{i+1, i}(1)$ for all possible $i$ (see (2)). As in the case of odd characteristic, we also obtain every $R_{i j}(1)$.

Now,

$$
\left(R_{32}(1), J^{\prime}\right)=R_{31}(a)
$$

and

$$
\left(R_{13}(a), R_{32}\left(a^{3}\right)\right)=R_{12}\left(a^{4}\right)
$$

But squaring is an automorphism of a field of characteristic two, so $a^{4}$ is primitive if $a$ is. Hence, using equations (2) through (9), we obtain every rotation (ii) with

$$
R_{21}(a) J^{\prime}=T_{n n}(1)
$$

for any $y$ in $\mathrm{GF}(q)$,

$$
\left(R_{1 n}(y), T_{n n}(1)\right)=\left(\begin{array}{cc}
I & y E_{n 1}+y E_{1 n}+y^{2} E_{11} \\
0 & I
\end{array}\right) .
$$

Also

$$
\begin{aligned}
D^{-1} R_{n-1, n}(y) D & =T_{1 n}(y) ; \\
\left(R_{1 n}(y), T_{n n}(1)\right) T_{1 n}(y) & =T_{1}\left(y^{2}\right) .
\end{aligned}
$$

Again, since squaring is an automorphism, $y^{2}$ is arbitrary. The proof may now be completed as above.

5. The group $\operatorname{Sp}\left(6,2^{m}\right)$. In this section we show 
Lemma 4. $\operatorname{Sp}\left(6,2^{m}\right)$ is generated by $D$ and $J^{\prime}$ :

$$
J^{\prime}=\left(\begin{array}{cc}
I+a E_{21} & a^{-1} E_{33} \\
0 & I+a E_{12}
\end{array}\right),
$$

where $a$ is primitive in $\mathrm{GF}(q)$.

Computing $A_{1}=D^{-1} J^{\prime} D, A_{2}=D^{-2} J^{\prime} D^{2}, A_{3}=\left(J^{\prime}, D\right)$, we get

$$
D^{-1} A_{2} D^{-1} A_{2} D^{-1} A_{3} D^{3}=R_{13}\left(a^{2}\right)
$$

and $D^{-1} R_{13}\left(a^{2}\right) D=T_{12}\left(a^{2}\right)$. Then since $D^{-1}\left(R_{13}(x), A_{1}\right)^{2}=R_{13}\left(x^{2}\right)$, we get every $R_{13}\left(a^{2^{k}}\right), k>0$. Now $a^{q}=a$ is some power of two, so we get the matrix $R_{13}(a)$. We have shown that the matrix $T_{12}\left(a^{2}\right)$ can be obtained from $D$ and $J^{\prime}$. Assume that we have obtained the matrix $T_{12}\left(a^{k}\right)$. But then, because

$$
\left(R_{13}(a), D^{-1} T_{12}\left(a^{k}\right) D\right)=T_{12}\left(a^{k+1}\right),
$$

we can get every matrix of the form $T_{12}(x)$, for any $x$ in $\operatorname{GF}(q)$. Hence we have shown that every $R_{13}(x)$, for any $x$ in $\mathrm{GF}(q)$ is obtained from $D$ and $J^{\prime}$.

Now we compute $A_{4}=\left(R_{13}\left(a^{2}\right), A_{1}\right), A_{5}=\left(D^{-1} R_{31}\left(a^{2}\right) D\right)\left(D^{-2} R_{31}\left(a^{4}\right) D^{2}\right) A_{4} A_{2}$, $A_{6}=R_{13}(1) R_{31}(1) D^{-2} A_{5} D^{2} R_{31}(1) R_{13}(1), A_{7}=\left(R_{13}(1), A_{6}\right)$, and finally $\left(D^{-1} A_{7} D, R_{13}(x)\right)=R_{23}(y x)$, with $y=a^{-1}+a^{5}$. If the field is neither $\mathrm{GF}(2)$ or $\mathrm{GF}(4), y \neq 0$ and $x$ can be chosen so that $y x=a$. Then the group generated by $D$ and $J^{\prime}$ contains every rotation (ii) with $\operatorname{det} U=1$.

$$
R_{21}(a) J^{\prime}=T_{3}\left(a^{-1}\right) \text {. }
$$

Hence, the proof may now be completed as before.

Now consider the case of the field GF(4). GF(4) is generated over GF(2) by a root of $1+x+x^{2}$. If $a$ is a primitive element then the three nonzero elements are $1, a, 1+a=a^{2}=a^{-1}$. We shall now show that the group $\operatorname{Sp}(6,4)$ is generated by the matrix $D$ and the matrix $J^{\prime}$ defined by

$$
J^{\prime}=\left(\begin{array}{cc}
I+a E_{21} & a E_{33} \\
0 & I+a E_{12}
\end{array}\right)
$$

First,

$$
\begin{aligned}
\left(J^{\prime}, D^{-1} J^{\prime} D\right) & =R_{31}\left(a^{2}\right), \\
D^{-3} R_{31}\left(a^{2}\right) D^{3} & =R_{13}\left(a^{2}\right) .
\end{aligned}
$$

Moreover,

$$
\begin{aligned}
& \left(D^{-2} R_{31}\left(a^{2}\right) D^{2}\right)\left(D^{-3} J^{\prime} D^{3}\right)\left(D^{-1} R_{31}\left(a^{2}\right) D\right) \\
& \left(R_{13}(1), D^{-1} J^{\prime} D\right)\left(D^{-2} J^{\prime} D^{2}\right)=\left(\begin{array}{cc}
I & 0 \\
a^{-1} E_{22} & I
\end{array}\right) .
\end{aligned}
$$


Then $T_{3}\left(a^{-1}\right)$ is a conjugate of this matrix by $D$ and

$$
\begin{aligned}
R_{13}(1) T_{3}\left(a^{-1}\right) R_{13}(1) D^{2} T_{3}\left(a^{-1}\right) D^{-2} & =T_{13}\left(a^{-1}\right), \\
D^{-1} T_{13}\left(a^{-1}\right) D & =R_{21}\left(a^{-1}\right) .
\end{aligned}
$$

Now surely $a^{-1}$ is primitive if $a$ is, so we may proceed as before.

The case of $\mathrm{GF}(2)$ is best handled in [7] where Room exhibits two generators for the group $\operatorname{Sp}(6,2)$, one of which has period two.

6. The main theorem. We have thus far seen that the group $\operatorname{Sp}(2 n, q)$ has two generators for $n>2$. We are now in a position to prove the theorem of the introduction.

For $q$ a power of two, the matrix $J^{\prime}$ of $\S \S 4$ and 5 have period two. For $q$ odd the matrices $D$ as above and $J$ defined by

$$
J=\left(\begin{array}{cc}
I+b E_{12}-2 E_{22} & 0 \\
0 & I+b E_{21}-2 E_{22}
\end{array}\right),
$$

where $b=a / 2$, a primitive, generate the symplectic group.

Among the conjugates of $J$ and $D$ are the following

$$
\begin{aligned}
& J_{1}=\left(\begin{array}{cc}
I+b E_{21}-2 E_{22} & 0 \\
0 & I+b E_{12}-2 E_{22}
\end{array}\right), \\
& J_{2}=\left(\begin{array}{cc}
I+b E_{32}-2 E_{33} & 0 \\
0 & I+b E_{23}-2 E_{33}
\end{array}\right) .
\end{aligned}
$$

Then,

$$
\left(J, J_{1}, J_{2}\right)=R_{32}(x),
$$

where $x=b^{3}+4 b$, and the commutator $(X, Y, Z)$ is defined to be $(X,(Y, Z))$. Also,

$$
\left(J, J_{1} J_{2} J_{1}\right)=R_{32}(y)
$$

where $y=b^{3}+2 b$. Now,

$$
R_{32}(x)\left(R_{32}(y)\right)^{-1}=R_{32}(x-y)=R_{32}(2 b)=R_{32}(a),
$$

and this matrix, together with $D$ are known generators.

In the natural map $\operatorname{Sp}(2 n, q)$ onto $\operatorname{PSp}(2 n, q), D$ and $J^{\prime}$ are mapped onto generators, and the coset containing $J$ has period two.

\section{REFERENCES}

1. A. A. Albert and John Thompson, Two element generation of the projective unimodular group, Illinois J. Math. 3 (1959), 421-439.

2. H. R. Brahana, Pairs of generators for the known simple groups of order less than one million, Ann. of Math. (2) 31 (1930), 529-549. 
3. - Regular maps and their groups, Amer. J. Math. 49 (1927), 266-284.

4. L. E. Dickson, The theory of linear groups, Dover, New York, 1958.

5. J. Dieudonné, Sur les groupes classiques, Actualités Sci. Ind. No. 1040, Hermann, Paris, 1958.

6. L. K. Hua and I. Reiner, On the generators of the symplectic modular group, Trans. Amer. Math. Soc. 65 (1949), 415-426.

7. T. G. Room, The generation by two operators of the symplectic group over $\mathrm{GF}(2), \mathrm{J}$. Austral. Math. Soc. 1 (1959), 38-46.

8. T. G. Room and R. J. Smith, A generation of the symplectic group, Quart. J. Math. Oxford Ser. (2) 9 (1958), 177-182.

9. R. Steinberg, Generators for simple groups, Canad. J. Math. 14 (1962), 277-283.

UNIVERSITY OF CHICAGO,

Chicago, Illinois

INSTITUTE FOR DEFENSE ANALYSES,

Princeton, New Jersey 